\title{
Advanced Artificial Electronic Skin Based pH Sensing System for Heatstroke Detection
}

\author{
Ganesh Kumar Mani ${ }^{1, *}$ and Yuka Nimura ${ }^{2}$, Kazuyoshi Tsuchiya ${ }^{1,3}$ \\ ${ }^{1}$ Micro/Nano Technology Center, Tokai University, 4-1-1 Kitakaname, Hiratsuka, \\ Kanagawa, 259-1292 Japan. \\ ${ }^{2}$ Graduate School of Science and Technology, Tokai University, 4-1-1 Kitakaname, \\ Hiratsuka, Kanagawa, 259-1292 Japan. \\ ${ }^{3}$ Department of Precision Engineering, Tokai University, 4-1-1 Kitakaname, Hiratsuka, \\ Kanagawa, 259-1292 Japan.
}

Table S1: ECR Sputtering Condition

\begin{tabular}{|c|c|c|}
\hline Parameter & \multicolumn{2}{|c|}{ Condition } \\
\hline Target & Ti & Au \\
\hline Flow rate of Ar gas [sccm] & 0.8 & 0.8 \\
\hline Sputtering time [min] & 1 & 5 \\
\hline Accelerato r [eV] & 2500 & 2500 \\
\hline Micro wave power [W] & 100 & 100 \\
\hline${\text { Target angle }\left[{ }^{\circ}\right]}^{\text {Target offset }[\mathrm{mm}]}$ & 45 & 45 \\
\hline
\end{tabular}

Ti and Au sputtering was used for making contact electrodes for measurements. 
Table S2: RF Sputtering Condition

\begin{tabular}{|c|c|c|c|c|}
\hline Factor & \multicolumn{4}{|c|}{ Condition } \\
\hline Target & $\mathrm{Ag}$ & $\mathrm{AgIO}_{3}$ & $\mathbf{S b}$ & $\mathrm{Sb}_{2} \mathbf{O}_{3}$ \\
\hline Flow rate of Ar gas $[\mathbf{s c c m}]$ & $\mathbf{5 0}$ & $\mathbf{5 0}$ & $\mathbf{5 0}$ & $\mathbf{5 0}$ \\
\hline Sputtering time $[\mathrm{min}]$ & $\mathbf{5}$ & $\mathbf{6 0}$ & $\mathbf{1 5}$ & 15 \\
\hline Pressure $[$ Pa] & 1 & 3 & 1 & 1 \\
\hline Distance from target $[\mathrm{mm}]$ & $\mathbf{8 0}$ & $\mathbf{4 0}$ & $\mathbf{8 0}$ & $\mathbf{8 0}$ \\
\hline Micro wave power $[\mathbf{W}]$ & $\mathbf{5 0}$ & $\mathbf{1 0}$ & $\mathbf{3 0}$ & $\mathbf{5 0}$ \\
\hline
\end{tabular}

Reference $\left.(\mathrm{Ag} / \mathrm{AgIO})_{3}\right)$ and working electrode $\left(\mathrm{Sb} / \mathrm{Sb}_{2} \mathrm{O}_{3}\right)$ sputtering conditions.

\section{Supplementary Figure S1}

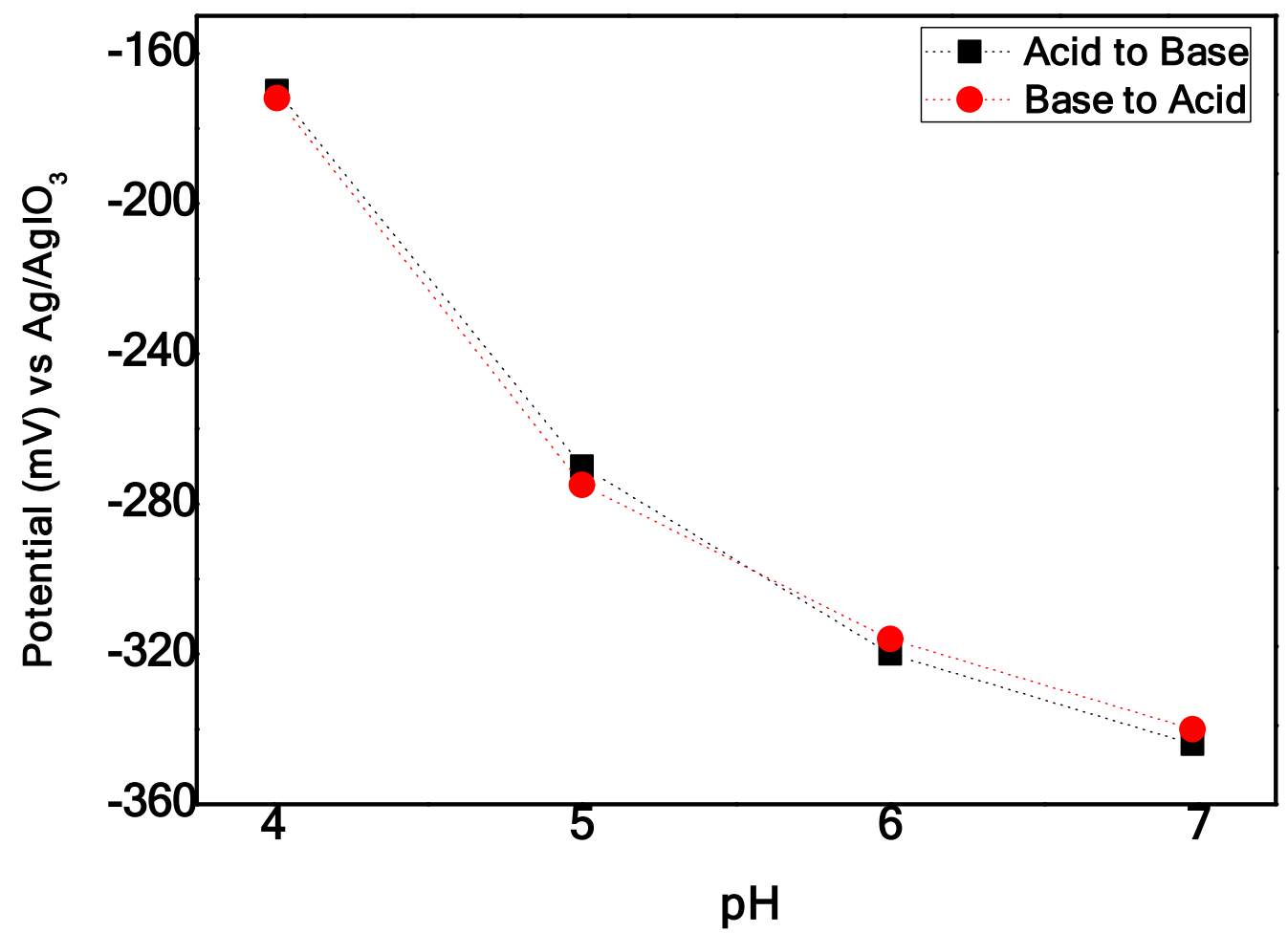

The sensor was tested for its memory effect. Hence, the sensing was performed from acid to base condition and base to acid condition. However, the memory effect was negligible. 


\section{Supplementary Video: V1}

Twisting and bending the flexible nanosheet sensor pasted on the artificial forearm video is also provided. 